\title{
Social and Active Inclusion of the Elderly in the City Through Affective Walkability
}

\author{
Stefania Bandini ${ }^{1,2} \mathbb{D} \cdot$ Francesca Gasparini $^{1}$
}

Received: 30 June 2021 / Accepted: 7 September 2021 / Published online: 15 October 2021

(c) The Author(s) 2021

\begin{abstract}
The development of cities aware of the needs of all citizens is a priority, especially in the case of elderly people. In this context, particular attention should be paid to the analysis of walkability. It has been proved that performing walking activities produces significant benefits both for physical and mental health, above all in elderly subjects. Besides traditional criteria adopted to evaluate walkability, we here propose a novel approach defined affective walkability. Being able to interpret the emotions of elderly walking in the urban environment and interacting with vehicles and other citizens, it is possible to evaluate if and to which extent an environment is perceived safe, comfortable and walkable. One way to obtain quantitative measures of walkability is to assess safety perception relying on physiological signals that can be considered indicators of emotions and mood. The assessment of affective walkability requires the design and performance of rigorous experiments to properly collect data. In this paper, the proposal of an affective walkability is presented and the developed experimental protocols, their performance and preliminary results are illustrated.
\end{abstract}

Keywords Affective walkability $\cdot$ Physiological signals $\cdot$ Elderly

This research is supported by the FONDAZIONE CARIPLO "LONGEVICITY-Social Inclusion for the Elderly through Walkability" (Rif. 2017-0938) and by the Japan Society for the Promotion of Science (Ref. L19513).

Stefania Bandini

stefania.bandini@unimib.it

Francesca Gasparini

francesca.gasparini@unimib.it

1 Department of Informatics, Systems and Communication, University of Milano-Bicocca, 20126 Milan, Italy

2 RCAST, Research Center for Advanced Science and Technology, The University of Tokyo, Tokyo, Japan 


\section{Introduction}

The phenomenon of urbanization is a global trend of the 21 st Century, requiring governments and institutions to design and plan the development of cities strongly considering the quality of life of the citizens. At today, over half of the world's population lives in urban areas and great part of the inhabited world areas are expected to urbanize further over the coming decades: in numbers, by 2030 over $60 \%$ of the global population will live in cities and large urban agglomerates [18]. Urbanization shift is occurring constantly and globally since 2007 , joined to the increasing proportion of elderly in the population, because of the population ageing, due to longevity and a decline in both mortality and fertility rates. Population ageing is occurring fastest in high and developed countries, being Italy and Japan at the first places experiencing an ageing society [35]. Moreover, world demographic projections show that, by 2030, the number of people aged 65 and over will be the $25 \%$ of the population, and many of them will live in urban areas. This trend makes the activities of city planners and managers very challenging, due to rising demand of services and infrastructures for the elderly citizens as reported in Fig. 1.

The World Health Organization (WHO) [25] recognized trends convergence of ageing and urbanization, introducing the concept of Age-friendly Cities, a conceptual framework addressing urban development towards an active ageing of the population. Within this framework, many countries gave their adhesion to adopt recommendations through the development of policies supporting pedestrian mobility fostering an active inclusion of aged citizens living the urban environment. The investigation of innovative solutions to enhance the development of pedestrian areas and, in general, to promote walking in urban territories is becoming a mandatory requirement for the design of future cities. Enhancing pedestrian mobility represents, in fact, a key element for guaranteeing the social participation and inclusion of the elderly citizens. The European Charter of Pedestrian Rights (1988) highlighted the need to ensure the comfort and safety of pedestrians in urban areas, including the elderly and people with impaired mobility:
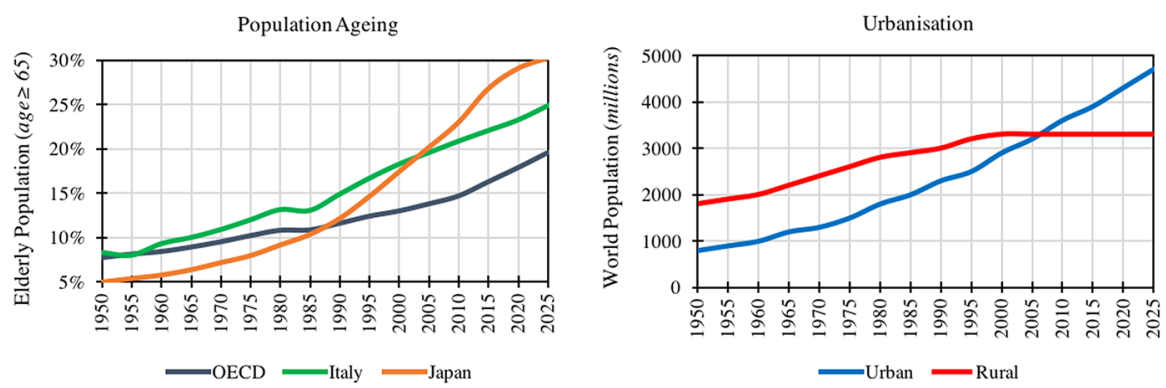

Fig. 1 Demographic projections of population ageing [35] and and urbanization trends [18] 
- Art. I- "The pedestrian has the right to live in a healthy environment and freely to enjoy the amenities offered by public areas under conditions that adequately safeguard his physical and psychological well-being";

- Art. III - "Children, the elderly and the disabled have the right to expect towns to be places of easy social contact and not places that aggravate their inherent weakness".

From here, advanced urban planning activities [34] shift towards the focus on the concept of walkability [2], namely how friendly the urban environment is for walking, guaranteeing the comfort and safety of vulnerable road users while walking (e.g., barrier-free streets, maintenance of sidewalk, visibility of road signs, public places for outdoor activities) [17].

The term walkability refers to those design elements that guarantee the accessibility, comfort and safety of the urban setting for walking [12]. Jeff Speck [32] recently proposed a General Theory of Walkability, which explains the essential elements for evaluating the level of walkability of urban environments: (i) presence of services within a walkable distance; (ii) level of comfort and safety experienced by people while walking; (iii) attractiveness of the urban areas in terms of architectural design and social context.

The methodological framework offered by Jeff Speck allows considering the measurement of basic indicators, and suggests a deeper investigation of feelings and perceptions of elderly citizen living their mobility in the city (ii). Following this research line and within the project "LONGEVICITY: Social Inclusion for the Elderly through Walkability" [20] this work aims at developing innovative research focusing on the affective walkability [3]. The idea behind affective walkability is that it is possible to measure the affective state of pedestrian and in particular of the elderly to study if and to which extent an environment is perceived safe, comfortable and walkable. A walking environment that is elderly-friendly is a priority while planning the design of the cities of the future as well as to improve the existing ones [13]. Besides more conventional self-assessment questionnaires, affective walkability relies on physiological responses, which are uncontrolled and autonomous reactions of human nervous system, considered honest indicators of emotions and mood, and thus nowadays widely adopted to recognize affective states, and in particular to quantify the level of stress suffered by the subjects while performing a certain task. One way to obtain quantitative measures of walkability is to assess safety perception while moving within an urban environment, in particular while walking, crossing and in general interacting with vehicles and other pedestrians. The assessment of safety perception can be performed with experiments and observations designing the experimental setting in a controlled environment as well as with data collections in the real world setups. Nowadays wearable devices, and smartphones easily integrate several sensors that measure physiological signals [36], making the acquisition of this data more comfortable and usable in day-life activities, even in case of elderly people. Physiological signals are already widely used in the emotion and affect recognition fields, having proved their effectiveness and usefulness in this area $[6,15]$. They have a recent wide application for interpreting and analyzing user's sleep behavior [29] as well as for daily activities recognition [36] and in e-health 
application for monitoring and remote assistance [24]. As a consequence, these signals could be easily adopted as valid indicators to assess quantitatively the safety perception of the elderly, while interacting with the surrounding environment.

The paper is organized as follows. Firstly, the purpose and approach of the mentioned LONGEVICITY project is illustrated in Sect. 2. Then a description of the walkability assessment criteria that, taking into account the needs of the elderly inhabitants, can guide the definition of proper experiments is reported in Sect. 3 and introduces the description of data collection and methods for the affective walkability assessment presented in Sect. 3. The paper concludes with final remarks about results and future works.

\section{The LONGEVICITY Project}

In the context of progressive urbanization [1] and population ageing [35] global trends, the project "LONGEVICITY - Social Inclusion for the Elderly through Walkability" has the objective to study advanced solutions to sustain the social inclusion of the elderly in urban contexts by enhancing their pedestrian mobility. According to the Age-friendly City framework [25], the project aims at developing innovative strategies to sustain the active ageing of the citizens by enhancing the walkability [32] of the City of Milan (Italy), with reference to the level of usefulness, comfort, safety, attractiveness and accessibility of urban environments.

The LONGEVICITY project is based on a strongly cross-disciplinary research approach, integrating skills, methodologies and tools ranging from Social Sciences, Design of Services, Artificial Intelligence and Complex Systems Science. The international team is composed by four main partners: University of Milano-Bicocca (Department of Computer Science, Systems and Communication), Politecnico di Milano (Department of Design), The University of Tokyo (Research Center for Advanced Science and Technology), and AUSER Volontariato Lombardia.

The research plan of the project is structured in work packages, whose main aims are: (i) assessing the level of walkability of the City of Milan (Italy) through advanced GIS analyses and adopting affective state analysis to collect the perception of safety and comfort; (ii) setting up of the living labs where to execute a series of outdoor and indoor activities participated by a large sample of elderly citizens (e.g., walking groups, participatory design activities, storytelling activities to preserve the memory of the chosen areas); (iii) empirically investigating age-driven pedestrian mobility through questionnaires, field observations, controlled experiments and simulations.

The project is based on methodological and computational tools aimed at achieving solutions considering the needs and perceptions of senior citizens with respect to infrastructures and mobility services in the City of Milan. The LONGEVICITY project is hence characterized by a methodological interplay between advanced tools for social research, combined with approaches for the development of innovative technologies. The cities of the future will be characterized, in fact, by the growing presence of long-lived/active citizens, and it will then be necessary to design technologically advanced infrastructures and services to provide support to them, and 
to experiment new technological frameworks to collect data revealing the feeling of walking in an urban environment.

\section{Main Criteria to Evaluate Walkability for the Elderly}

The walking behavior of aged pedestrians is mainly conditioned by the physiological progressive decline in the operation of: (i) perceptive sensors (e.g., limited perception of light and colors, inability to tune out background noise) and (ii) motor-cognitive skills (e.g., reduced range of motion, loss of muscle strength and coordination, changes in posture, diminished attention and reaction time, spatial disorientation).

Such bodily changes, in the majority of cases, make elderly people walking slower than adults, and lead to a subjective perception of physical vulnerability and a sense of fragility at the psychological level. The main set of walkability assessment criteria focused on the needs of walking elderly could be listed as follows:

- Usefulness Urban areas should be designed to guarantee the presence of many and diverse public services for the elderly at a walkable distance from their place of residence (e.g., land-use mix; street connectivity; transport services; social and health care service; commercial activities).

- Comfort Urban areas should be designed to accommodate the comfort of the elderly while walking (e.g., pavement type; continuity on side-walks; installation of ramps for people with reduced mobility; urban furniture for resting; green areas with trees, benches, tables and fountains).

- Safety Urban areas should be designed to guarantee the safety of elderly pedestrians while walking and crossing (e.g., absence of barriers and pothole on sidewalks; speed bumpers; traffic lights; illumination systems in proximity of the zebra crossing; legible horizontal and vertical signage).

- Attractiveness Urban areas should be designed to have a polycentric structure, with several and distinctive areas of attraction for the elderly inhabitants (e.g., points of interest, amenities, public spaces and events; quality of the architectural streetscape; vitality of the social context).

- Legibility Urban areas should be designed to be legible, memorable and navigable, to enable the elderly to easily locate themselves and navigate through the city (e.g., roads toponymy; legible road signs; place-based maps for indicating public services).

In the LONGEVICITY project, a wide range of methods have been studied to empirically measure the level of pedestrian friendliness of urban environments, within a multi-disciplinary approach (e.g. urban studies, architecture, urban sociology, environmental psychology, computer science):

- Geographic information systems [5, 33, 37] providing analytical tools for structured geo-referred data about the topographical, infrastructural and architectural elements of a urban area and the census indicators of the socio-demographical characteristics of the inhabitants. 
- Field observations [10, 12, 30] to annotate the behavior characteristics of the elderly while walking and crossing through a determined urban area, to produce a behavioral map by means of manual coding or video tracking analysis.

- Audit tools [7, 8, 23] applied as self-report scales or questionnaires for collecting qualitative and quantitative data about the elderly subjective perception of the level of pedestrian friendliness of urban areas.

- Web applications $[9,33]$ to collect opinions of the elderly about to rate the level of walkability of an urban area using mobile applications. For instance RateMyStreet, Walk Score and Walkanomics are the most popular web applications for walkability assessment.

- Social media data [4, 26, 27] achieve a bottom-up characterization of the level of walkability of urban areas, directly based on the geo-referred contents generated by the elderly users on social media.

- Computer-based simulations [11, 19, 38] provide optimized architectural solutions for managing urban areas, thanks to the possibility to simulate elderly pedestrian dynamics and to test alternative conditions and courses of action.

To provide innovative approaches in the measurement of the level of pedestrian friendliness of urban environments, we introduce an affective walkability assessment to quantitatively evaluate the safety and comfort perception of elderly people while moving within the city. The next section will illustrate the methods we have developed and the results obtained that pave the way towards the definition of novel criteria to evaluate walkability that takes into account the perception of the elderly living the urban environment.

\section{Affective Measurements of Walkability}

Measuring and recognizing the affective state of citizens during walking activities contribute to a better comprehension of their perception of the environment, and a better definition of walkable urban area. Quantitative assessment of affective walkability passes through the measurement of the level of stress affecting pedestrians in their interaction with the urban environment, in particular while walking and road crossing, and in general during interaction with vehicles and others pedestrians. Among all the possible stress sources, the interaction of a subject with the environment is one of the most significant, and can be interpreted as a defensive reaction activated to protect oneself from dangerous events [31]. Measuring physiological signals using wearable sensors is the research direction we adopted to grasp more knowledge about pedestrian safety perception. This novel approach stimulates the design of experimental affective walkability assessment. It is fundamental to investigate which physiological characteristics can be adopted to reveal affective states and how these should be processed to have a clearer and more comprehensive recognition of stress levels [22]. There are several factors that contribute to change the perception of stress in a subject, including age, sex, disability and cognitive impairment. Moreover, other variables deriving from the environment should be considered, such as the presence of loud noises, bulky work vehicles, poor visibility of 
oncoming cars (e.g. due to cars parked on the side of the road) and lack of signs that facilitate the crossing [21]. To assess innovative approaches towards affective walkability the definition of proper experimental settings and protocols is mandatory. In particular, we need to study how human affective state changes with respect to space varying conditions and not only with respect to a time varying stimulus, and thus we consider as a proper external stimulus the spatial variation of the environment where the human being is moving. To this end we have designed two different types of experiments involving both young adults and elderly people:

- In-vitro experiments The data collection is carried out in a controlled laboratory environment. In this case the interference with external stimuli are limited and data quality is higher. The disadvantage of these experiments relies in the non-real nature of the stimuli.

- In-vivo experiment The data collection is performed in a real-life environment, that has the advantage of inducing more realistic affective states, but introduces several source of noise, lowering data quality.

In-vitro and in-vivo experiments have complementary aspect of data quality while considering data acquisitions that intent to capture subjective responses, such as affective states and safety perception. In-vitro experiments permit to control the environmental conditions, reducing the noise in data due to the interference with several external stimuli, besides the ones we are interested in, as well as reducing uncontrolled events. However, we pay this noise attenuation with a reduction of the reliability of the acquired data, that are not representative of real-life conditions, and can in general correspond to more attenuated responses. On the other hand, in-vivo experiments permit to capture the effective subjective responses to real-life stimuli. However, it is not always easy to recognize and disentangle the interference with disturbing events, that are not objects of our investigation. We thus rely in data analysis that both consider data acquired in controlled and uncontrolled environment, to benefit of their complementary positive aspects.

All the experimental protocols hereafter described were approved by the Ethics Committee of the University of Tokyo (in-vitro experiment) and of the University of Milano-Bicocca (in-vivo experiment).

\subsection{Subjects Involved}

To focus on how elderly people perceive the interaction with the urban environment, two distinct group of subjects are taken into consideration in both the two types of experiments: the elderly, over the age of 60 and a control group of young adults, with an age between 18 and 35 years. The inclusion criteria can be summarized as follows:

1. absence of major medical disorders (in particular neurological and severe cognitive disorders); 
2. no pharmacological therapy, that could interfere with the collected data (such as drugs, or anti-depressants);

3. no significant visual impairment (normal or corrected to normal visual acuity);

4. no significant hearing impairment;

5. autonomous mobility without supports.

\subsection{Physiological Signals for Affective Walkability Assessment}

In our investigations we collect several physiological signals as well as inertial data. Physiological signals, such as Photoplethysmogram (PPG) and Galvanic Skin Response (GSR) are well indicated to detect emotional arousal, related to sensory alertness, mobility, and readiness to respond, activated in the interaction between subjects and the environment as a defensive reaction to preserve safety.

Moreover, dealing with a dynamic interaction, motion data both physiological, measuring the muscle activity with Electromyogram (EMG) and inertial (accelerometer and gyroscope data) are considered. The sensors adopted in the experiments are shown in Fig. 2.

Relying on different signal sources that register both physiological and dynamic walking responses will provide accurate results for affective state recognition tasks, in a multi-modal approach.

\subsection{Experimental Settings}

The final aim of data acquisition through properly designed experiments is to correlate physiological signals with walking activities in heterogeneous environments, thus understanding how a person is feeling while he or she moves through a given
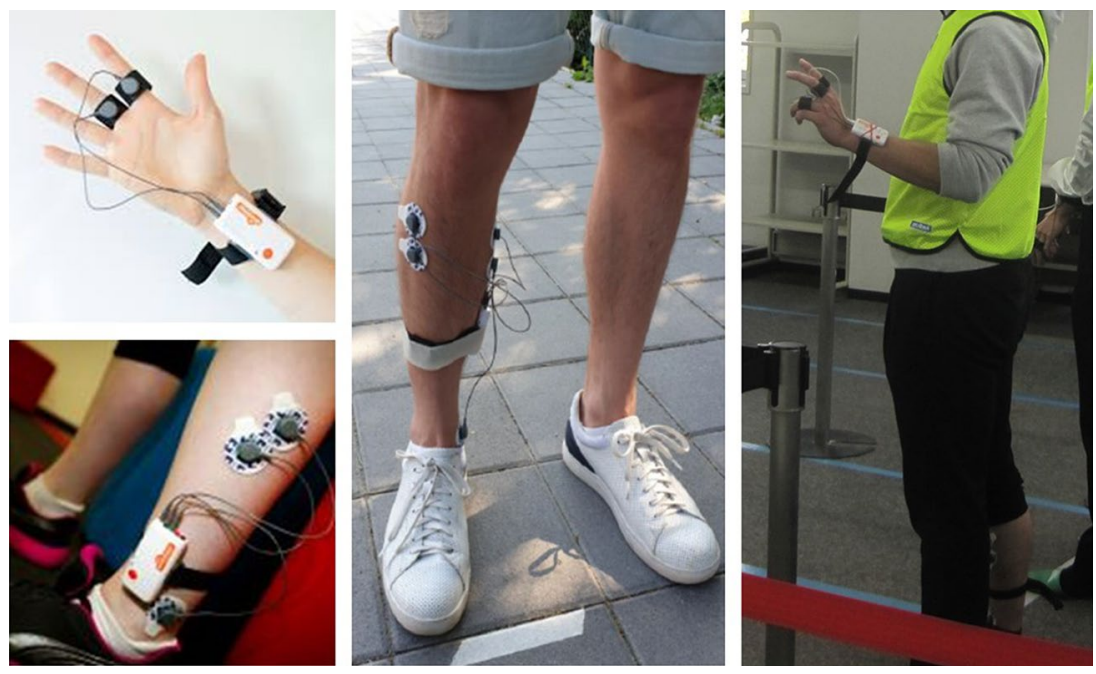

Fig. 2 Wearable devices adopted both in-vitro and in-vivo experiments 
setting. The two different types of experiments we have designed and carried out to acquire valuable data are described in what follows.

\subsubsection{In-Vitro Experiment}

The first experiment has been carried out indoor, in a controlled environment, namely a designed and dedicated space at the RCAST-Research Center for Advanced Science and Technology, at the Komaba Campus of The University of Tokyo.

Three within subject conditions (free walk, forced speed walk, and collision avoidance) have been proposed in one experimental session, performed by two experimental groups: a population of young adults, composed of 16 Japanese master and $\mathrm{PhD}$ students, with average age of 24.7 years (standard deviation $=3.3,4$ women) and a group of 20 Japanese elderly subjects (retired) with average age of 65.15 (standard deviation $=2.7,10$ women). The protocol of the experiment, that lasts about $30 \mathrm{~min}$, can be summarized as follows:

1. Free walk (FW) Two subjects at the same time can walk freely without obstacles or speed constraints along the path depicted in Fig. 3, back and forth.

2. Collision avoidance Two subjects at the same time walk with their own pace along the path clockwise and counterclockwise respectively. At about half of the path, they reach the collision avoidance zone where they have to avoid the collisions with both the obstacles (swinging pendulum) and the other subject.
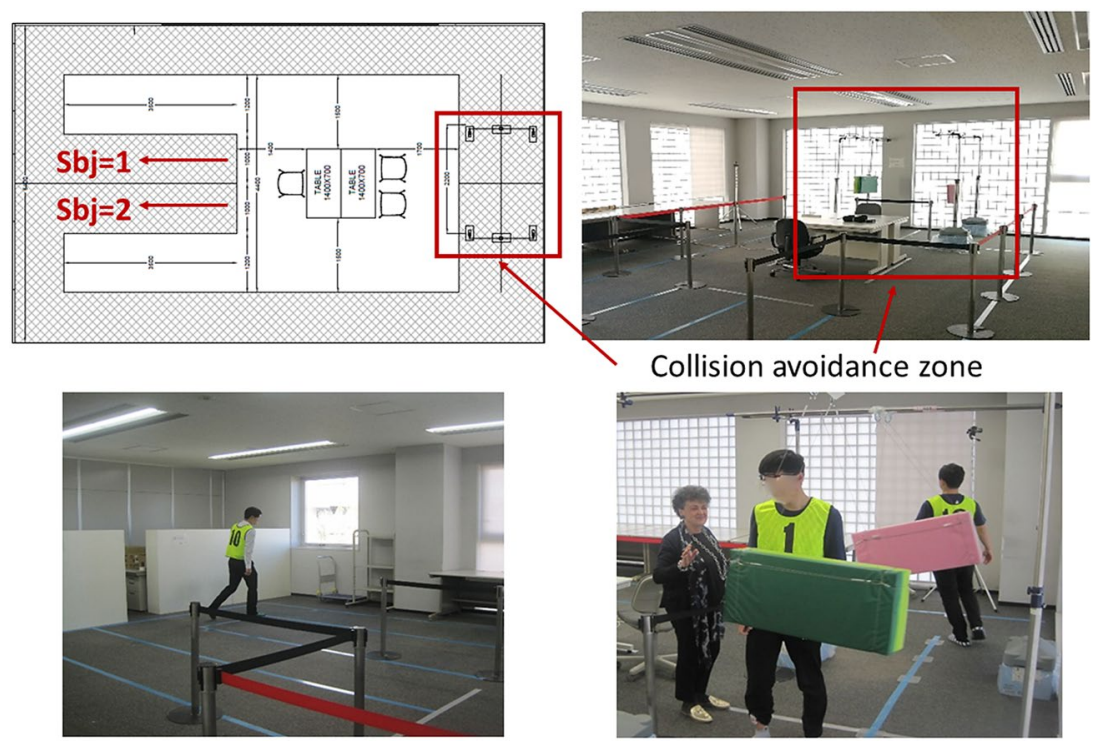

Fig. 3 Controlled experiment. Top left: the plant of the laboratory, where the path chosen for the walking activities is reported. A red rectangle identifies the collision avoidance zone in the two images of the first raw. In this zone, two obstacles are moved by one of the experimenter and the two subjects have to avoid the collision (figure bottom right). During the rest of the path, subjects walk with their own natural pace 
Then they complete the path, with their natural pace and go back in the opposite direction repeating the same actions. This task is thus composed of a free walking activity approaching and leaving the obstacle zone: WO, and the effective avoiding collision action, Obs.

3. Forced speed walk The two participants walk with a forced speed based on the metronome ticking, along the same path. Three speeds are considered: F1 $=70$ $\mathrm{bpm}, \mathbf{F} 2=85 \mathrm{bpm}$ and $\mathbf{F 3}=100 \mathrm{bpm}$. At the end, a questionnaire is provided to the participants to obtained information about the preferred walking frequency among those constrained by the metronome.

All the tasks are separated by a period of resting time called Baseline acquisition (BL) of about $1 \mathrm{~min}$, to gather reference physiological responses. The whole procedure is repeated three times.

\subsubsection{In-Vivo Experiment}

The experiment considers two different walking scenarios related to different perception of safety: free walking on a sidewalks, and crossing a two way road in correspondence to a zebra crossing, without traffic lights. Without becoming dangerous for the subjects who undergo the experiment, the more dangerous the crossing, the greater the emotional arousal triggered and the better the signals acquired.

The whole protocol is described as follows:

1. Initial Baseline (B): 2 min session to acquire the reference physiological signals, where the subject has to stay straight up and still.

2. Self assessment questionnaire on self-esteem (Q). [28].

3. Experiment Core: repeated 4 times

- Walking on sidewalk (W) (non-stressful task).

- $60 \mathrm{~s}$ baseline (B) recording, also intended to bring the subject back to a neutral state before the next task.

- Crossing the road back and forth $(\mathbf{C})$ (stressful task).

- $60 \mathrm{~s}$ baseline (B), same as before.

- Self-assessment questionnaire $(\mathbf{Q})$ to evaluate the level of safety perception of each crossing.

\section{End of trial}

Within the experiment core, the order of the walking and crossing tasks are randomly selected for each subject, to avoid possible biases, introduced by repeating the same task order. The whole experiment lasts about 20 min. In Fig. 4 the experimental protocol is reported.

We have carried out the experiment described by the protocol depicted in Fig. 4 two times, hereafter called sessions, considering two different populations and geographical places. 


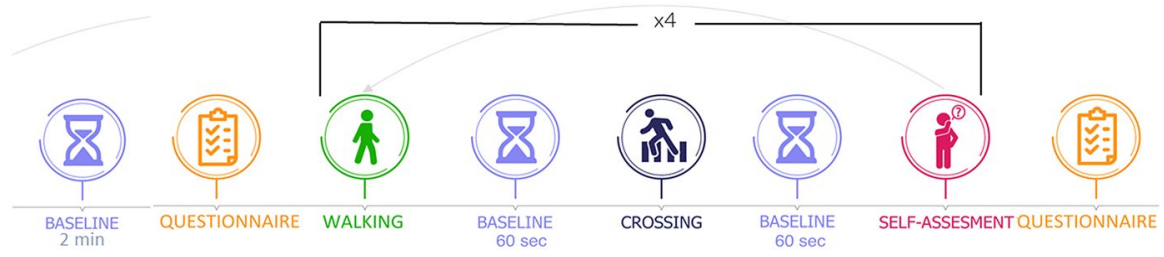

Fig. 4 The In-Vivo Experimental Protocol

In the first session we have involved 14 young adults, 7 males and 7 females, aged between 20 and 26 years $($ mean $=24.42$, standard deviation $=1.65$ ), all computer science students at the University of Milano-Bicocca. The chosen environment contemplated an unsupervised crossroad on a two-way road, not far from the main buildings of the University of Milano-Bicocca, depicted in Fig. 5, on the left.

This crossing is considered moderately dangerous for pedestrians for the following reasons:

- The crosswalk is located on a very busy road, given its location near offices, the university and a shopping mall.

- The crosswalk is unsupervised, with no traffic lights for neither the cars or the pedestrians.

- The numerous parking lots positioned alongside the intersection limit the view of the pedestrian before and while crossing the road.

- Different vehicles travel alongside this road, ranging from bicycles to cars, trucks and buses.
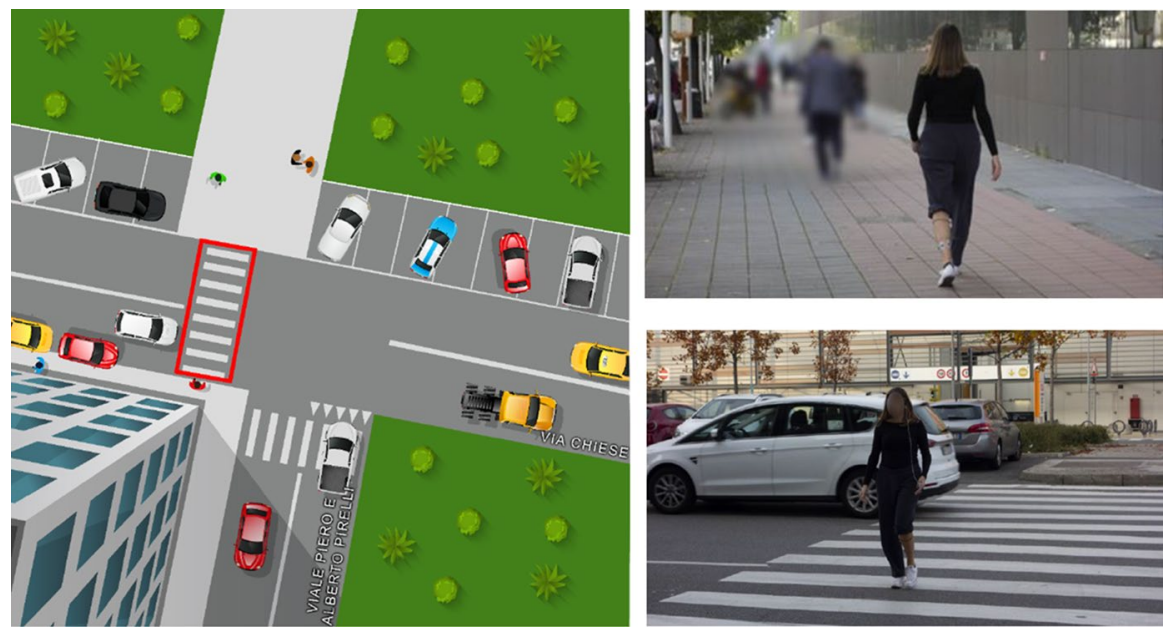

Fig. 5 The chosen intersection with the selected crossroads highlighted in red on the left. Two of the subjects involved in walking on the sidewalk (top right) and crossing the intersection (bottom right) 
In the second session we have involved 21 elderly people, citizen of Cantù. Cantù is a Lombard city that has about 40,000 inhabitants and is located at the foot of the Como pre-Alps. The history of Cantù is very ancient and has several narrow streets often uphill. However, this difficult configuration of the city does not prevent heavy vehicles, buses and a large number of cars from engaging the streets of the city center, which is very busy at rush hour, as illustrated in Fig. 6. All these characteristics make Cantù an ideal setting to test stressful routes or crossings for an older person.

\subsection{Experimental Results}

The raw signals obtained during the data acquisitions described in the previous session, were filtered, and pre-processed. Proper features were evaluated on GSR, PPG and EMG pre-processed signals and considered to perform statistical inference and to feed machine learning algorithms. To deepen the topic for the in-vitro experiment, please refer to [16] while refer to [14] for the in-vivo data acquisition and process. The obtained results can be summarized as follows:

1. All the performed experiments have confirmed that different walking conditions, such as crossing in the presence of vehicles or walking on the sidewalk, on one side or interacting with an obstacle or walking with different paces, can be related to different safety perception, and induced different physiological reactions in subjects.

As an example of the variations of the physiological signals with respect to the task, we report in Fig. 7 a subject's physiological responses for the entire duration of the in-vivo experiment. For the labels in the figure, please refer to Sect. 4.3.2. Every performed task can be easily distinguished along the physiological responses. In particular the muscle activity, acquired by the EMG signal (bottom red line), underlines differences among walking and crossing tasks and
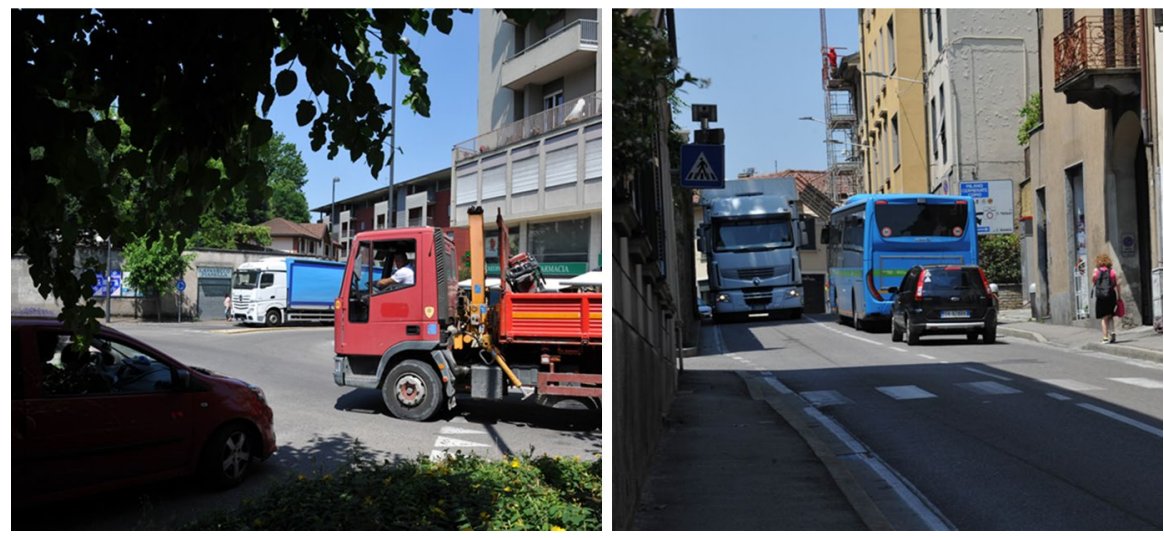

Fig. 6 Examples of heavily trafficked roads in the center of Cantù 


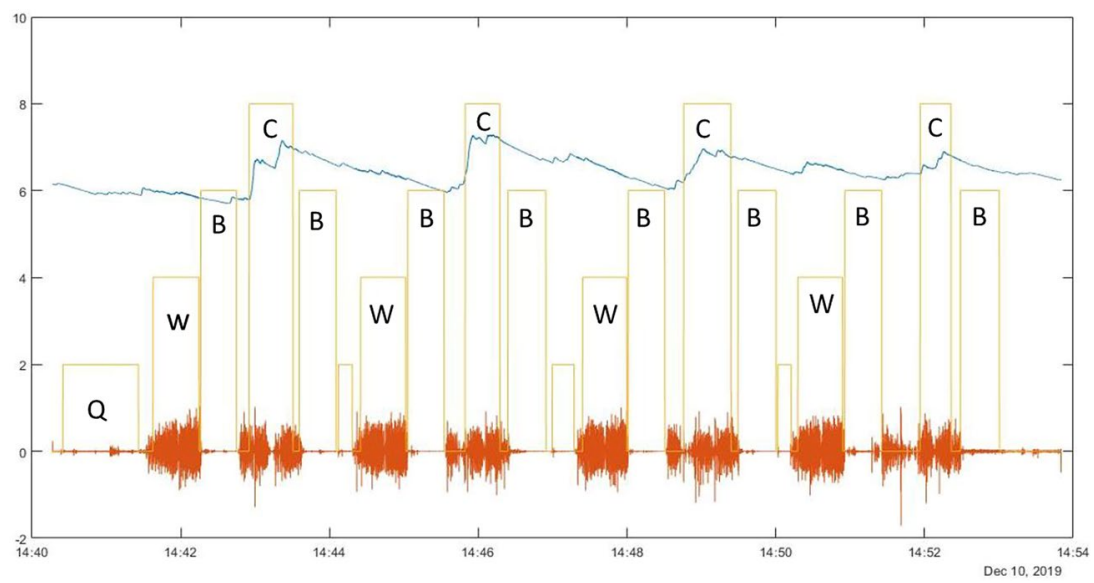

Fig. 7 The plot reports the physiological signals of a single participant in the in-vivo experiment. The orange track depicts the EMG signal and the blue one the GSR signal. Q indicates the Questionnaire task (missing in the smallest windows), $\mathrm{W}$ the sidewalk activity, $\mathrm{B}$ the baseline acquisitions and $\mathrm{C}$ the crossing tasks

periods of standing and baseline. On the other hand, the GSR peaks and activation in skin conductivity (top blue line) clearly correspond to the crossing task.

2. From data analysis we have observed that arousal is activated when interacting with the environment and can be adopted as a measure of stress considered as a defensive reaction to protect oneself from dangerous events.

3. Safety perception and subjects behaviour can be different with respect to age. In particular analysis of the physiological signals confirmed that elderly people are used to keep a more careful behavior than the youngest ones in case of collision avoidance tasks.

As the collected data do not follow normal distributions, we apply the Kruskal Wallis statistical test, which is a non-parametric version of classical one-way ANOVA, that compares the medians of the groups of data given as input to determine if the samples come from the same population (or, equivalently, from different populations with the same distribution).

In Fig. 8 the energy variations of the leg muscle activities analyzed in the invitro experiment are reported, comparing the two populations while performing the same task, in particular during collision avoidance task (figure on the left) and immediately after (figure on the right). A p-value lower than 0.001 has been obtained for both the comparisons. From Fig. 8 it emerges that while approaching the obstacle (figure on the left) in case of the elderly, the energy in general decreases, while in the case of young adults it increases, confirming a more careful behaviour of the elderly. After crossing the collision avoidance zone, on the other hand, the elderly tend to increase their speed while the young adults tend to decelerate to come back to their previous speed.

Moreover, from the analysis of the estimated pace frequency during the forced speed tasks (in-vitro experiment), we have observed that the elderly people strug- 

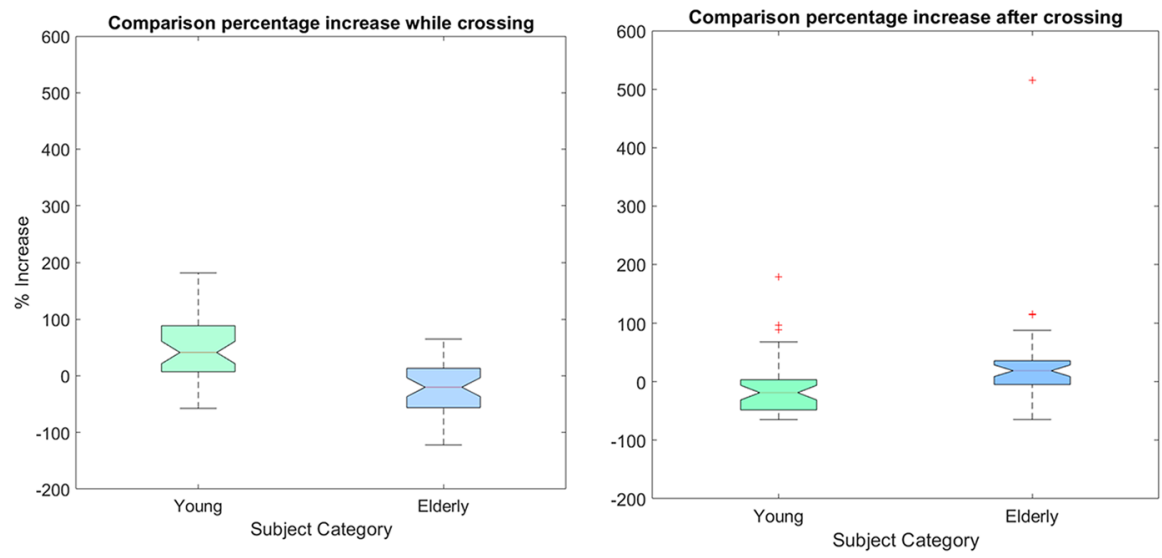

Fig. 8 Boxplots reporting the increase of muscle energy detected in the two population analyzed (young adults and elderly) while crossing the collision avoidance zone (on the left) and after that crossing (on the right). A positive value corresponds to an increase in muscular energy, while a negative percentage represents a decrease in the energy value. The line in the center of the boxplot represents the median value

gle more than young adults to respect the metronome forced speeds. In particular, this behaviour has been observed mainly in the two lower speeds. In Table 1, the accuracy of the two population groups in following the three different metronome frequencies, obtained analyzing the activity of the leg muscles, are reported.

From all the performed analyses it emerges that the two populationgroups would need a more specific and in-depth analysis to create ad-hoc environments able to meet their needs. The results reached with these analyses suggest that affective walkability could be considered as a novel field of research to better understand the age-related behaviour of subjects in real-world stressful urban environments.

Table 1 Accordance in percentage between the computed pace frequencies and the metronome frequencies, evaluated with respect to the two muscle activities on the leg (columns) and the two considered populations (rows)

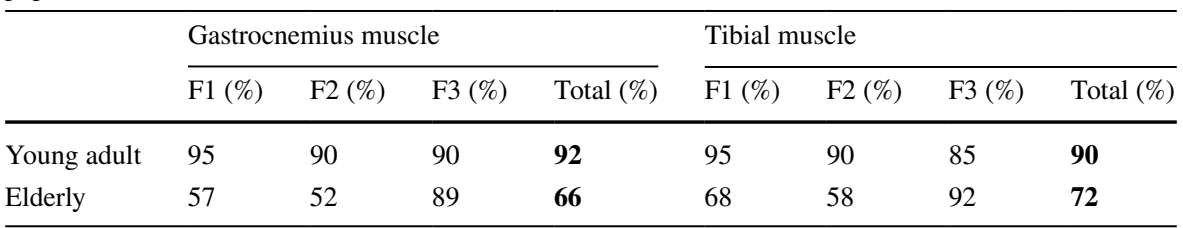

The accordance is reported with respect to the three different metronome frequencies (F1, F2 and F3) and on average in column Total, which is shown in bold. 


\section{Conclusions}

The need to develop novel methods to collect and analyze data from walking activities is growing to provide suggestions and recommendations in the design of more walkable cities. Due to the parallel growing of ageing population and urbanization phenomena, supporting active ageing means not only the application of walkability assessment through more traditional methods, but also by considering affective perceptions. In the framework of the LONGEVICITY project, a novel approach has been developed, based on the collection of physiological data coming from wearable sensors during the performance of ad-hoc in-vivo and in-vitro experiments, considering both young and elderly people. The paper illustrated the designed experimental protocols, their application and preliminary meaningful results to support an affective walkability. Starting with these preliminary results, more data acquisition are required to consolidate our findings, in particular to better distinguish between different populations (young adults and elderly). Physiological responses are highly subjective, and even in the case of the same stimulus perception, they can significantly vary within subjects. Thus a special effort should be devoted in normalizing data to better perform population based analysis. Finally, results coming from self-assessment questionnaires such as The Big Five Personality Traits questionnaire should be included to better profile affective responses of each subject. Furthermore, new data will allow the adoption of machine learning approaches including deep learning. The future of the research will proceed towards the design of computer-based simulation programs considering affective states in the behavior of virtual pedestrians, to create complex urban scenarios where the study of vehicle-pedestrian dynamics during road crossing. The fall of the research results will be addressed towards decision makers to improve and ameliorate the promotion of safe and walkable cities through the introduction of new methods to evaluate the degree of walkability by considering affective perceptions.

Acknowledgements We want to give our thanks to Prof. Katsuhiro Nishinari and his staff (partner of the LONGEVICITY project) for their indispensable support during the in-vitro experiment held at RCAST - The University of Tokyo. Our thanks also to Marta Giltri, Alessandra Grossi and Gianluca Toffanin for their valuable contributions in collecting and analyzing data, and to all the elderly people and students that actively participated to the experiments.

Funding Open access funding provided by Università degli Studi di Milano - Bicocca within the CRUICARE Agreement.

\section{Declarations}

Conflict of interest The authors declare that they have no conflict of interest.

Open Access This article is licensed under a Creative Commons Attribution 4.0 International License, which permits use, sharing, adaptation, distribution and reproduction in any medium or format, as long as you give appropriate credit to the original author(s) and the source, provide a link to the Creative Commons licence, and indicate if changes were made. The images or other third party material in this article are included in the article's Creative Commons licence, unless indicated otherwise in a credit line to the material. If material is not included in the article's Creative Commons licence and your intended use is 
not permitted by statutory regulation or exceeds the permitted use, you will need to obtain permission directly from the copyright holder. To view a copy of this licence, visit http://creativecommons.org/licen ses/by/4.0/.

\section{References}

1. Ageing in cities-policy highlights. (2015). OECD Publishing, Paris. https://doi.org/10.1787/ 9789264231160-en.

2. Abley, S., \& Hill, E. (2005). Designing living streets-a guide to creating lively, walkable neighbourhoods, Transport Research International Documentation, Transport Research Board.

3. Bandini, S., \& Gasparini, F. (2020). Towards affective walkability for healthy ageing in the future of the cities. In: Proc. 5th Workshop on Artificial Intelligence for Ambient Assisted Living (AIxIA 2019), vol. 2559. CEUR-WS.

4. Berzi, C., Gorrini, A., \& Vizzari, G. (2017, July). Mining the social media data for a bottomup evaluation of walkability. In: International Conference on Traffic and Granular Flow (pp. 167-175). Springer, Cham.

5. Blečić, I., Cecchini, A., Congiu, T., Fancello, G., \& Trunfio, G. A. (2015). Evaluating walkability: a capability-wise planning and design support system. International Journal of Geographical Information Science, 29(8), 1350-1374, Taylor \& Francis.

6. Can, Y. S., Arnrich, B., \& Ersoy, C. (2019). Stress detection in daily life scenarios using smart phones and wearable sensors: A survey. Journal of Biomedical Informatics, 92, 103139.

7. Cerin, E., Saelens, B. E., Sallis, J. F., \& Frank, L. D. (2006). Neighborhood environment walkability scale: validity and development of a short form. Medicine and Science in Sports and Exercise, $38(9), 1682$.

8. Day, K., Boarnet, M., Alfonzo, M., \& Forsyth, A. (2006). The Irvine-Minnesota inventory to measure built environments: development. American Journal of Preventive Medicine, 30(2), $144-152$.

9. Duncan, D. T., Aldstadt, J., Whalen, J., \& Melly, S. J. (2013). Validation of walk scores and transit scores for estimating neighborhood walkability and transit availability: a small-area analysis. GeoJournal, 78(2), 407-416.

10. Ewing, R., Hajrasouliha, A., Neckerman, K. M., Purciel-Hill, M., \& Greene, W. (2016). Streetscape features related to pedestrian activity. Journal of Planning Education and Research, 36(1), 5-15.

11. Feliciani, C., Crociani, L., Gorrini, A., Vizzari, G., Bandini, S., \& Nishinari, K. (2017). A simulation model for non-signalized pedestrian crosswalks based on evidence from on field observation. Intelligenza Artificiale, 11(2), 117-138.

12. Forsyth, A. (2015). What is a walkable place? the walkability debate in urban design. Urban Design International, 20(4), 274-292.

13. Gaglione, F., Cottrill, C., \& Gargiulo, C. (2021). Urban services, pedestrian networks and behaviors to measure elderly accessibility. Transportation Research Part D: Transport and Environment, 90, 102687.

14. Gasparini, F., Giltri, M., \& Bandini, S. (2021). Safety perception and pedestrian dynamics: Experimental results towards affective agents modeling. AI Communications (Preprint), 1-15.

15. Gasparini, F., Giltri, M., \& Bandini, S. (2020). Discriminating affective state intensity using physiological responses. Multimedia Tools and Applications, 1-21.

16. Gasparini F., Grossi A., Nishinari K., Bandini S. (2021) Age-Related Walkability Assessment: A Preliminary Study Based on the EMG. In: Baldoni M., Bandini S. (eds) AIxIA 2020 - Advances in Artificial Intelligence. AIxIA 2020. Lecture Notes in Computer Science, 12414, 423-438, Springer, Cham. https://doi.org/10.1007/978-3-030-77091-4_25.

17. Gehl, J. (2013). Cities for people. Island press.

18. Heilig, G.K. (2012). World urbanization prospects: the 2011 revision. United Nations, Department of Economic and Social Affairs (DESA), Population Division, Population Estimates and Projections Section, New York, vol. 14. 
19. Helbing, D., Jiang, R., \& Treiber, M. (2005). Analytical investigation of oscillations in intersecting flows of pedestrian and vehicle traffic. Physical Review E, 72(4), 046130.

20. https://sites.google.com/unimib.it/longevicity/home.

21. Kim, H. (2020). Wearable sensor data-driven walkability assessment for elderly people. Sustainability, 12(10), 4041.

22. Kim, J., Ahn, C. R., \& Nam, Y. (2019). The influence of built environment features on crowdsourced physiological responses of pedestrians in neighborhoods. Computers, Environment and Urban Systems, 75, 161-169.

23. Lee, S., \& Talen, E. (2014). Measuring walkability: a note on auditing methods. Journal of Urban Design, 19(3), 368-388.

24. Nath, R.K., \& Thapliyal, H. (2021). Wearable health monitoring system for older adults in a smart home environment. In 2021 IEEE Computer Society Annual Symposium on VLSI (ISVLSI) (pp. 390395). IEEE.

25. Organization, W.H. (2007). Global age-friendly cities: A guide. World Health Organization.

26. Quercia, D., Aiello, L.M., Schifanella, R., \& Davies, A. (2015). The digital life of walkable streets. In Proceedings of the 24th international conference on World Wide Web (pp. 875-884).

27. Quercia, D., Schifanella, R., \& Aiello, L.M. (2014). The shortest path to happiness: Recommending beautiful, quiet, and happy routes in the city. In Proceedings of the 25th ACM conference on Hypertext and social media (pp. 116-125). https://doi.org/10.1145/2631775.2631799.

28. Rosenberg, M. (1962). The association between self-esteem and anxiety. Journal of Psychiatric Research, 1(2), 135-152. https://doi.org/10.1016/0022-3956(62)90004-3.

29. Sano, A., \& Picard, R. W. (2014). Comparison of sleep-wake classification using electroencephalogram and wrist-worn multi-modal sensor data. In 2014 36th Annual International Conference of the IEEE Engineering in Medicine and Biology Society (pp. 930-933). https://doi.org/10.1109/EMBC. 2014.6943744.

30. Singh, R. (2016). Factors affecting walkability of neighborhoods. Procedia-Social and Behavioral Sciences, 216, 643-654.

31. Sioni, R., \& Chittaro, L. (2015). Stress detection using physiological sensors. Computer, 48(10), 26-33.

32. Speck, J. (2013). Walkable city: How downtown can save America, one step at a time. Macmillan.

33. Tsiompras, A.B., \& Photis, Y.N. (2017). What matters when it comes to "walk and the city"? defining a weighted gis-based walkability index. Transportation research procedia, 24, 523-530.

34. Wefering, F., Rupprecht, S., Bührmann, S., \& Böhler-Baedeker, S. (2013). Guidelines. developing and implementing a sustainable urban mobility plan. In Workshop, Mar (p. 117).

35. World population ageing. (2013). United Nations, Department of Economic and Social Affairs, Population Division, Population Estimates and Projections Section, New York.

36. Yetisen, A. K., Martinez-Hurtado, J. L., Ünal, B., Khademhosseini, A., \& Butt, H. (2018). Wearables in medicine. Advanced Materials, 30(33), 1706910.

37. Yin, L. (2017). Street level urban design qualities for walkability: Combining $2 d$ and $3 d$ GIS measures. Computers, Environment and Urban Systems, 64, 288-296.

38. Zeng, W., Chen, P., Nakamura, H., \& Iryo-Asano, M. (2014). Application of social force model to pedestrian behavior analysis at signalized crosswalk. Transportation Research Part C: Emerging Technologies, 40, 143-159.

Publisher's Note Springer Nature remains neutral with regard to jurisdictional claims in published maps and institutional affiliations. 